\title{
Complementary Specific Pelvic Sequences on Routine Lumbar Magnetic Resonance Imaging Scans: An Imaging-Based Study Focused on Piriformis Syndrome
}

\author{
Selim AYHAN ${ }^{1,2}$, Vugar Nabi NABIYEV ${ }^{3}$, Yasin YETISYIGIT², Selcuk PALAOGLU², Mehmet YORUBULUT ${ }^{4}$ \\ ${ }^{1}$ Acibadem Mehmet Ali Aydinlar University, Vocational School of Health Sciences, Istanbul, Turkey \\ ${ }^{2}$ ARTES Spine Center at Acibadem Ankara Hospital, Department of Neurological Surgery, Ankara, Turkey \\ ${ }^{3}$ ARTES Spine Center at Acibadem Ankara Hospital, Department of Orthopaedics and Traumatology, Ankara, Turkey \\ ${ }^{4}$ Acibadem Ankara Hospital, Department of Radiology, Ankara, Turkey
}

Corresponding author: Mehmet YORUBULUT m.yorubulut@gmail.com, selim.ayhan@acibadem.edu.tr

\section{ABSTRACT}

AIM: To discuss the rationale and merit of specific pelvic sequences as an adjunct to routine lumbar magnetic resonance imaging (MRI) for early detection of piriformis syndrome (PS) and to disclose its frequency in patients who underwent radiological evaluation.

MATERIAL and METHODS: This retrospective, imaging-based study included all individuals who underwent lumbar MRI and those who were further evaluated with a pelvic MRI, but excluded all high-energy trauma cases. The patients' demographics and radiological features were reviewed using electronic patient records and hospital-based picture image archiving and communication system.

RESULTS: Overall, 1321 individuals (659 females; 662 males) underwent lumbar MRI during the study period, and of these, 485 (238 females; 247 males) were further analyzed with a pelvic MRI for differential diagnosis. Forty patients (8.2\%) (23 females; 17 males) were diagnosed with PS-all confirmed with MR neurography (MRN). On re-evaluation of all lumbar and pelvic MRls and MRNs, we realized that adding just three specific pelvic sequences to routine lumbar MRI scans were enough to accurately delineate the piriformis muscle pathologies and sciatic nerve intensities. The calculated frequencies of PS in females, males, and the whole study population within 57 months were $3.49 \%, 2.57 \%$, and $3.03 \%$, respectively.

CONCLUSION: Although PS being a clinical diagnosis, this study indicated that in patients whom PS was not suspected at the initial examination, $8.2 \%$ of them were finally diagnosed after MRI evaluations. Performing specific pelvic sequences as an adjunct to routine lumbar MRI can salvage underdiagnosed patients with PS and facilitate early detection of this pathological condition.

KEYWORDS: Back pain, Magnetic resonance imaging, Magnetic resonance neurography, Piriformis syndrome, Sciatica

\section{INTRODUCTION}

$\mathrm{T}$ The piriformis muscle (PM) is situated deep in the gluteal region and runs diagonally from the lower spine to the upper surface of the femur $(4,18)$. The PM is innervated by the spinal nerves L5 to S2 and primarily aids in external rotation of the hip joint in the extended thigh, abduction of the hip joint in the flexed thigh, and hip flexion during walking $(4,19,28,30,32)$. The PM is surrounded by some anatomical structures that either pass through or underneath it, of which the most crucial one is the sciatic nerve, which typically exits the pelvis inferior to the muscle at the greater sciatic notch.

Piriformis syndrome (PS) causes low back and buttock pain and sciatica, which is a controversial, indistinct diagnosis, frequently unrecognized or misdiagnosed. In the absence of a clear definition, the term PS generally refers to pain that is caused by the entrapment of sciatic nerve by the PM at
Selim AYHAN (1D): 0000-0003-0153-3012 Vugar Nabi NABIYEV (1) : 0000-0002-2766-5083
Yasin YETISYIGIT (1) : 0000-0002-9681-162X Selcuk PALAOGLU (10): 0000-0003-4977-718X
Mehmet YORUBULUT (D) : 0000-0003-1747-685X 
the greater sciatic notch $(6,7,9,14,15,18,19,29,30,35)$. The clinical features of PS comprise two principal components: somatic and neuropathic $(21,32)$. The somatic pain is caused by abnormal conditions, pathologies, or even anatomical variations originating from the PM or involving it. On the other hand, neuropathic pain is caused by sciatic nerve irritation or compression. However, the symptoms can sometimes arise from the surrounding anatomical structures $(2,6,9,21,32)$.

PS has been considered a purely clinical diagnosis and often a diagnosis of exclusion (30), although there is still no gold standard for diagnosing it. Nonetheless, according to a recent systematic review by Hopayian and Danielyan, a quartet of symptoms comprising buttock pain, pain aggravated on sitting, external tenderness near the greater sciatic notch, and any PS sign (i.e., pain with increased PM tension) constitute the syndrome (19). Furthermore, all the necessary diagnostic procedures, electrodiagnostic tests, and radiologic investigations, including ultrasonography, computed tomography, and magnetic resonance imaging (MRI) need to be performed to rule out any spinal and extra-spinal causes of sciatica or hip and sacroiliac joint pathologies $(32,39)$. Obtaining an accurate diagnosis is crucial for providing adequate and timely treatment as well as prevention of unnecessary interventions. Delay in diagnosis may lead to pathologic conditions of the sciatic nerve, including chronic somatic dysfunction and other compensatory changes, such as paresthesia, hyperesthesia, and muscle weakness, which result in pain (6).

The research focused on PS gained momentum during the 2000s because of it being an elusive pathology with significant associated morbidity and being responsible for health-related productivity loss. However, the data in the literature are ambiguous owing to the various definitions, study methodologies, and population surveyed $(6,8,11,20,32)$. The main aim of this single-center study is to understand the potential role and the feasibility of promptly adding specific pelvic sequences to the routine lumbar MRI scans for early detection of PS in patients who underwent radiological evaluation for low back and buttock pain, and sciatica.

\section{MATERIAL and METHODS}

\section{Patient Population}

This cross-sectional, retrospective, imaging-based research reviewed all individuals who underwent lumbar MRI and those who were further evaluated with a pelvic MRI scan during the diagnostic process between April 2013 and December 2017. The exclusion criteria were high-energy trauma cases and the patients whose radiological evaluations were not done in the sequence given above. A flow chart of the study design and a case example are presented in Figures 1, 2A-D. The patients' demographics and radiological features were reviewed using electronic patient records and hospital-based picture archiving and communication system. The database was similar to the one that we've reviewed at our previous retrospective study (1). The study was approved by the institutional review board (ATADEK 2018-8/6).

\section{Imaging Studies}

All MRI procedures were performed using a 1.5 Tesla scanner (MAGNETOM Aera, Siemens, Erlangen, Germany). The routine MRI protocol in our center for lumbar spine includes sagittal T1-weighted (W) and T2W sequences, axial T2W sequence, and sagittal and coronal short tau inversion recovery (STIR) sequences. The routine pelvis MRI comprised precontrast axial T1W, T2W, T2W fat saturation (FAT SAT) and diffusion WI sequences; coronal T1W and STIR sequences; sagittal STIR sequences; and postcontrast T1W FAT SAT sequences in all three planes. If PM asymmetry was detected during pelvic MRI, an MR neurography (MRN) (axial T1W and axial, coronal, and nerve-oriented T2W FAT SAT sequences) was performed subsequently at the same diagnostic stage. The MRI images in multiplanar reconstructions were reformatted and measured using syngo.via.

\section{Statistical Analysis}

Data were expressed as mean values and range (minimummaximum) for continuous variables and as percentages for categorical variables. Analyses were performed using Microsoft Office Excel (2007) for Windows.

\section{RESULTS}

A total of 1321 individuals (659 females and 662 males) underwent lumbar MRI during the study period. Further evaluation with pelvic MRI was performed on 485 cases (238 females and 247 males), of which 40 (8.2\%) (23 females and 17 males) were diagnosed with PS. All individuals diagnosed with PS were confirmed to have the presence of nerve compression or abnormally increased intensity on MRN, which was performed immediately after pelvic MRI on detecting an asymmetrical PM.

The demographic characteristics of the study population and the distribution of patients by the age groups are summarized in Table I and Figure 3, respectively.

After evaluating the pelvic MRI scans and MRNs, we realized that just an additional three specific pelvic sequences, namely axial T1W, axial T2W FAT SAT, and coronal STIR performed as an adjunct to the routine lumbar MRI scans helped us to adequately assess the PM pathologies, sciatic nerve intensities, and their compositions.

The calculated frequencies of PS within 57 months in females, males, and the whole study population were $3.49 \%, 2.57 \%$, and $3.03 \%$, respectively.

\section{DISCUSSION}

This single-center study was designed to review and discuss the rationale and advantages of performing specific pelvic sequences as an adjunct to routine lumbar MRI scans for early detection of PS. Moreover, it helped to find the frequency of PS among the group of patients who underwent radiological evaluation in a tertiary care unit.

Diagnostic difficulties and lack of a consistent definition make PS challenging $(8,13,25,26)$. The earliest description of 

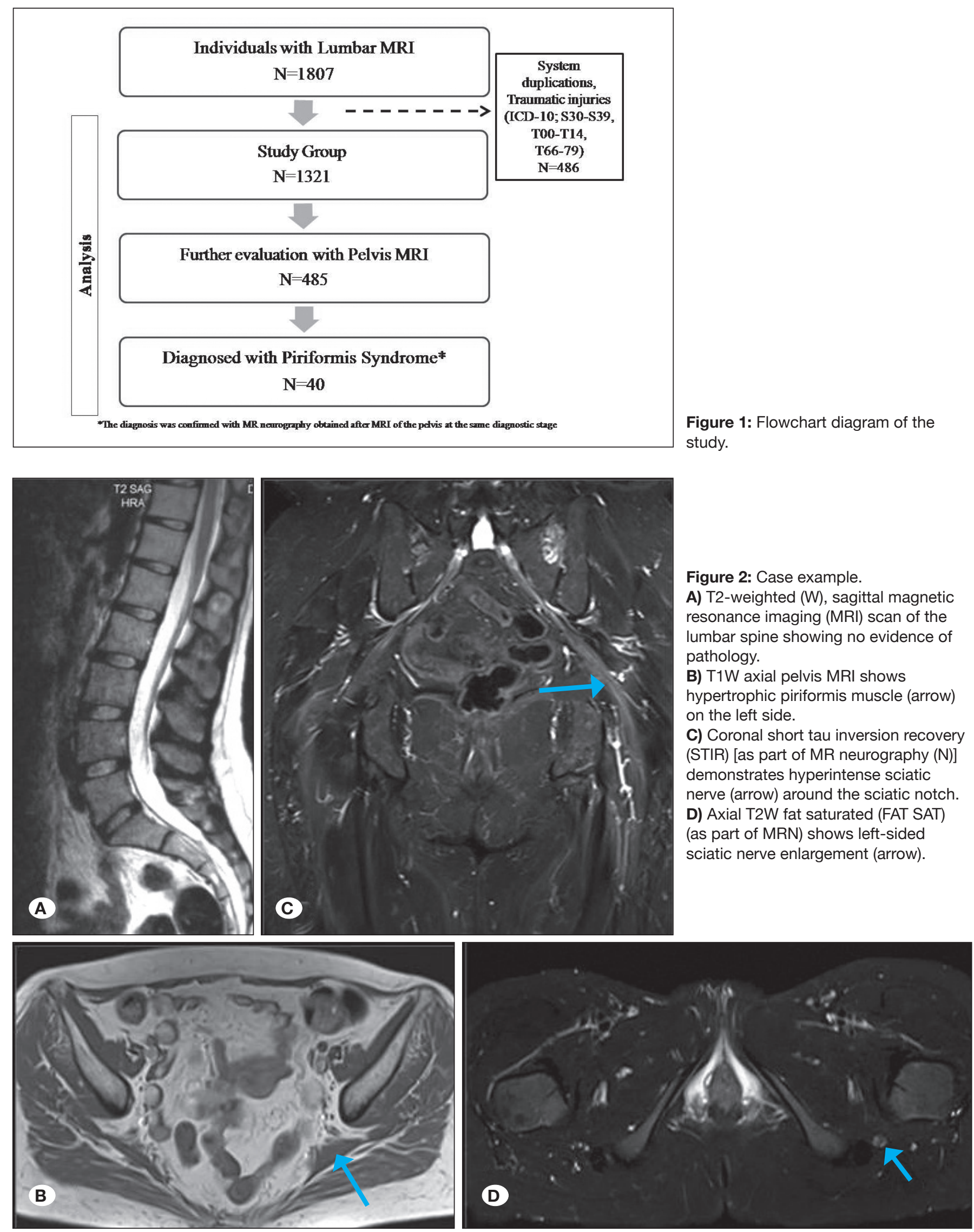

Figure 1: Flowchart diagram of the study.

Figure 2: Case example.

A) T2-weighted (W), sagittal magnetic resonance imaging (MRI) scan of the lumbar spine showing no evidence of pathology.

B) T1W axial pelvis MRI shows hypertrophic piriformis muscle (arrow) on the left side.

C) Coronal short tau inversion recovery (STIR) [as part of MR neurography (N)] demonstrates hyperintense sciatic nerve (arrow) around the sciatic notch. D) Axial T2W fat saturated (FAT SAT) (as part of MRN) shows left-sided sciatic nerve enlargement (arrow).
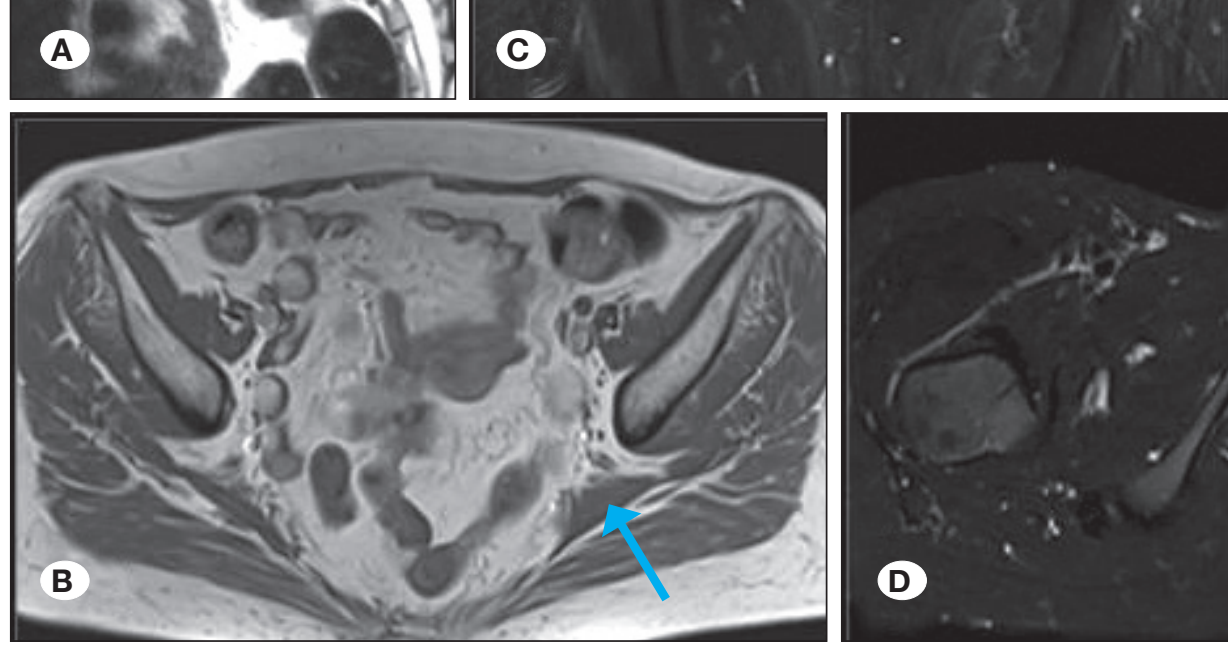
Table I: Demographic Characteristics of the Study Population

\begin{tabular}{lccc}
\hline & Lumbar MRI (+) patients & Further evaluated with Pelvic MRI & Patients with Piriformis Syndrome* \\
\hline Characteristic & $\mathbf{n}(\%)$ & $\mathbf{n}(\%)$ & $\mathbf{n}(\%)$ \\
\hline \begin{tabular}{l} 
Gender \\
\multicolumn{1}{c}{ Female }
\end{tabular} & $659(49.9)$ & & \\
\hline \multicolumn{1}{c}{ Male } & $662(50.1)$ & $238(49.1)$ & $\begin{array}{c}23(57.5) \\
\text { R: } 8, \text { L: } 15\end{array}$ \\
\hline $\begin{array}{l}\text { Total } \\
\text { Age, mean } \\
\text { (min-max)(years) }\end{array}$ & $1321(100)$ & $247(50.9)$ & $\begin{array}{c}17(42.5) \\
\text { R: 6, L: } 11\end{array}$ \\
\hline
\end{tabular}

*The diagnosis was confirmed with MR neurography obtained after MRI of the pelvis at the same diagnostic stage.

F: Female, M: Male, min: Minimum, max: Maximum, MRI: Magnetic resonance imaging, R: Right, L: Left

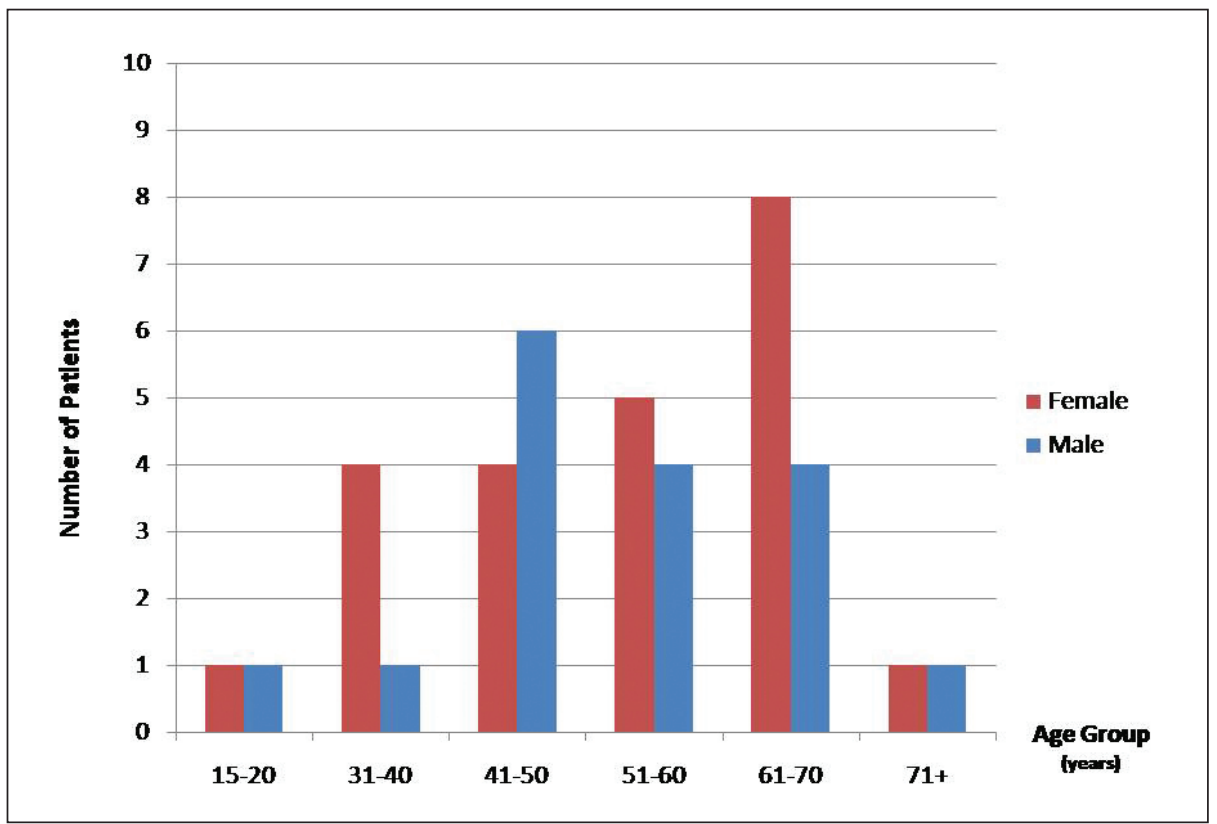

Figure 3: The patient distribution by age groups comprising 23 females and 17 males. this pathology, its evolution over time, and it getting termed specifically as "PS" can be found in the literature $(2,16,31,41)$.

According to the recent systematic review by Hopayian and Danielyan, PS is defined as buttock pain or sciatica caused by impingement of the sciatic nerve by the PM, diagnosed from a constellation of clinical signs and symptoms, which may be corroborated by MRI, endoscopic visualization, or response to treatment (19).

The results of epidemiological research on PS continues to remain ambiguous $(30,32,33)$ because of the difficulties mentioned above, as well as the successful first line treatment with nonsteroidal anti-inflammatory drugs in cases who have been treated as low back pain (LBP) patients (30), or local PM injection test being used as a diagnostic tool (27). The rate of incidence ranges from $0.3 \%$ to $6.25 \%$ for all cases of sciatica and $\operatorname{LBP}(8,34,39)$ with reported incidence rate of even $36 \%$
$(6,9,32)$. The calculated lifetime and annual occurrences of the condition in general population were $12.2 \%-27 \%$ and $2.2 \%-$ $19.5 \%$, respectively $(20,32)$. The syndrome occurs most frequently during the fourth and fifth decades of life, affecting individuals of all occupations and activity levels $(6,8,32)$. Notably, women are predisposed to this condition with the female-male (F:M) ratio being 3-6.4:1 (9,32,34).

Results of the present research were comparable to those reported in the literature. The calculated frequencies of PS in females, males, and the whole study population were $3.49 \%$, $2.57 \%$, and $3.04 \%$, respectively, during a 57 -month study period in the cohort of nondiscogenic patients. Most (47.5\%) of the patients were in the fourth and fifth decades of their lives, but we also noted that $30 \%$ of the patients in their sixties. Women had a higher incidence of this pathology with the F:M ratio being 1.35:1. We made the following assumptions during 
the research and after evaluating the results. First, at least some of the patients who were accurately diagnosed with PS on time would not be admitted to a tertiary center, and this would affect the frequency of cases. For this reason, we have not termed the detected frequency as "the incidence" of PS in this series. Second, we examined all patients who underwent lumbar MRI except those who were high-energy trauma cases, those with clearly discogenic causes, and those whose radiological examination was not performed in the sequence stated above. Nonetheless, some of these patients who were clinically diagnosed with PS at the time of clinical evaluation and lumbar MRI may not pursue further analysis, thereby affecting the calculated frequency of PS. Third, we assumed that those patients who further needed a pelvic MRI scan included those with probable PS or other mimics that cause pelvic pain, and regardless of the clinical examination performed at the time of lumbar MRI, apparently either the possibility of PS was not predicted or the mimics could not be excluded, thereby requiring further assessment. Nevertheless, each assessment stage causes loss of time and energy for both the patient and the healthcare provider and additionally increases the patient's anxiety.

The goals of paramount importance in the present study were to discuss the rationale for adding specific pelvic sequences to the routine lumbar MRI scans for accurate and timely diagnosis of PS and additionally hoping to increase the awareness regarding PS. Sciatica is a common pain problem frequently caused by lumbar disc herniation $(5,22)$. On the other hand, the most significant cause of nondiscogenic sciatica is the PS, which like disc herniation is equally prevalent as the cause of sciatica $(11,17,33)$. The routine MRI of the lumbar spine detects only the causes of sciatica originating from in and around the lumbar spine, thereby not detecting the extraspinal issues accurately. Therefore, pathologies may often be misdiagnosed $(10,22)$ and require further evaluation, including anatomical region-specific MRI or MRN. However, performing three specific pelvic sequences, namely :1) axial T1W, 2) axial T2W FAT SAT, 3) coronal STIR, as adjunct to the routine lumbar MRI scans would alert the requesting physician to look out for PS. Furthermore, the pelvic sequences would sufficiently demonstrate the PM pathologies, sciatic nerve intensities, and their compositions if indeed PS is present. Moreover, these adjunct sequences take no more than 5 minutes when added to a lumbar MRI and can provide crucial assistance for accurate and timely diagnosis of PS and/or its differential diagnosis. However, if any pathology other than $\mathrm{PM}$ and/or sciatic nerve is detected throughout the procedure, additional anatomical region-specific MRI and/or MRN studies should be performed.

PS is diagnosed clinically through the patient's history, physical examination, and exclusion of all other possible causes of the unilateral buttock and leg pain, such as lumbosacral degenerative diseases, intraspinal lesions, trochanteric bursitis, cysts and tumors around the sciatic notch, fibroids, endometriosis, and vascular pathologies $(6,18,35,40)$. However, one needs to be mindful that the "diagnosis of exclusion" is prone to misinterpretation, multiple causes for sciatica may coexist, and the presence of one cause does not exclude the other
(13). Once PS is clinically suspected $(3,6,12,19,23,25,32,40)$, specialized electrophysiological tests and imaging studies can be performed for accurate diagnosis $(6,8,12,32,38-40)$. Some researchers advocate the evaluation of the patients with clinical tests and performing a PM diagnostic block before performing electrophysiological or imaging studies, which are useful in excluding related pathologies $(6,27,32)$. Although a cross-sectional MRI of the pelvis or hip may be adequate in identifying PS $(37,38)$, MRN allows the detection of changes in nerve signal and architecture by revealing the nerve trajectory $(36,40)$. It also aids the physician to identify the structural modifications or pathologies of the PM. The advancements in imaging technology have recently enabled Wada et al. to present the diffusion tensor imaging and tractography of the sciatic nerve as an objective, effective, and supportive diagnostic tool for PS (39), albeit expensive and time-consuming.

Nonetheless, "PM asymmetry and associated unilateral hyperintense sciatic nerve" may raise a question about the normal values of these anatomical structures and the adequacy of the method for the detection of pathology. A well-documented fact through cadaveric studies is that the thickness and width of the sciatic nerve may differ (24), and it is apparent that these features are strongly affected by the individual's characteristics. Therefore, in our opinion, both the abnormally increased intensity and the asymmetry are the main features that helped us to evaluate PS. Additionally, our results are validated by a previous study that evaluated the patients diagnosed with nondiscogenic sciatica based on "no response to disc treatment or no evidence of disc herniation on lumbar MRI". The study found that the findings of PM asymmetry and unilateral sciatic nerve hyperintensity at the level of the sciatic notch on the imaging could distinguish the two distinct population of patients with a specificity of $93 \%$ for predicting good-toexcellent outcome from piriformis surgery; however, the sensitivity was $64 \%$ (11).

Our results would infer that if the clinician is aware of PS and communicates with the radiologists regarding the addition of the pelvic sequences at the time of clinical evaluation when discogenic causes are not convincing or excluded, then the whole process would eliminate further unnecessary evaluation time, energy, and bureaucracy until the PS is conclusively diagnosed. Indeed, in this study, PS was diagnosed solely by the specific stepwise radiologic methodology without any clinical information, and the authors were able to understand and demonstrate the reason why these patients were suffering. Therefore, we can conclusively suggest that adding the three specific pelvic sequences, namely axial T1W, axial T2W FAT SAT, and coronal STIR at the time of performing routine lumbar MRI scans would help in early detection of this pathologic condition.

Addition of specific pelvic sequences to routine lumbar MRI scans might be considered debatable in the context of relatively low PS incidence. We want to iterate that the values in our retrospective study that were calculated by the review of the MRI scans reflect the frequency for PS in a cohort of nondiscogenic patients and not the incidence at our private tertiary care setting, and may reflect those probably missing 
at the initial clinical examination. It should be borne in mind that incidence rates of up to $36 \%$ were reported $(6,10,35)$ with an accurate diagnosis not established in $80 \%$ of the total affected population with severe sciatica (12), especially more so in cases with concomitant lumbar disc herniation (14). Application of the new lumbar MRI protocol in appropriate clinical conditions may help guide future research to identify the incidence rate of concomitant disease as well.

Thanks to effective interdisciplinary collaboration, after evaluation of the present research results by our hospital council, we have started to perform the routine lumbar MRI protocols including the additional specific pelvic sequences mentioned above from the beginning of 2018. Our private tertiary care unit and referral spine and radiology center mostly deal with misdiagnosed diseases/patients and second and third opinions. However, nowadays, we can assess the differential diagnosis of lumbosacral radiculopathy in an appropriate and timely manner and often without the requirement of new MRIs and repeat outpatient appointments. An essential advantage in our center is the accessibility of both the radiologist and the clinician throughout all the imaging procedures. If a nonexpected pathology is detected during the procedure, the radiologist contacts the physician or vice versa, so that when necessary further detailed diagnostic investigations can be performed at the same diagnostic stage.

This study has several limitations. First, being a single-center study design in a private tertiary care setting might affect the study population and generalize the research results. Second, evaluating the radiological data without any clinical information to find out the incidence rates of PS, making it impossible to assess the clinicoradiologic-pathologic correlation. However, this does not mean that there is no need for clinical evaluation because based on clinical suspicion, all patients in the study who were finally diagnosed with PS, were evaluated using pelvic MRI and MRN for definitive diagnosis at the request of their treating physician or the radiologist. Third, there was no information on the previous treatment protocols. Fourth, the cost of adding pelvic sequences to the routine lumbar MRI scans was not calculated. Therefore, future research including a detailed statistical analysis and a cost-utility survey will be helpful.

\section{CONCLUSION}

Despite the calculated frequencies being relatively low in a cohort of nondiscogenic patients, this single-center retrospective study was found that performing specific pelvic sequences as an adjunct to routine lumbar MRI scans proved beneficial for both the patients and the physicians because of timely diagnosis of PS, which is a significant cause of disability. Accurate and timely diagnosis is critical in planning an individualized treatment course, avoiding unnecessary interventions, and improving the patient's quality of life, all of which can offer satisfaction to both the patient and the healthcare provider. Additionally, a cost-utility analysis should be performed.

\section{ACKNOWLEDGMENTS}

Preparation for publication of this article is partly supported by Turkish Neurosurgical Society.

\section{- REFERENCES}

1. Ayhan S, Nabiyev VN, Yorubulut M, Palaoglu S: Sacral stress fractures: An imaging based cross-sectional study. Journal of Turkish Spinal Surgery 29:237-241, 2018

2. Beaton LE, Anson BJ: The relation of the sciatic nerve and its subdivisions to the piriformis muscle. Anat Rec 70:1-5, 1937

3. Beatty RA: The piriformis muscle syndrome: A simple diagnostic maneuver. Neurosurgery 34:512-514; discussion 514, 1994

4. Benson ER, Schutzer SF: Posttraumatic piriformis syndrome: Diagnosis and results of operative treatment. J Bone Joint Surg Am 81:941-949, 1999

5. Bickels J, Kahanovitz N, Rubert CK, Henshaw RM, Moss DP, Meller I, Malawer MM: Extraspinal bone and softtissue tumors as a cause of sciatica. Clinical diagnosis and recommendations: Analysis of 32 cases. Spine (Phila Pa 1976) 24:1611-1616, 1999

6. Boyajian-O'Neill LA, McClain RL, Coleman MK, Thomas PP: Diagnosis and management of piriformis syndrome: An osteopathic approach. J Am Osteopath Assoc 108:657-664, 2008

7. Broadhurst NA, Simmons DN, Bond MJ: Piriformis syndrome: Correlation of muscle morphology with symptoms and signs. Arch Phys Med Rehabil 85:2036-2039, 2004

8. Cass SP: Piriformis syndrome: A cause of nondiscogenic sciatica. Curr Sports Med Rep 14:41-44, 2015

9. Dey S, Das S, Bhattacharyya P: Piriformis syndrome: A clinical review. Journal of Evolution of Medical and Dental Sciences 2:2502-2508, 2013

10. Dudeney S, O'Farrell D, Bouchier-Hayes D, Byrne J: Extraspinal causes of sciatica. A case report. Spine (Phila Pa 1976) 23:494-496, 1998

11. Filler AG, Haynes J, Jordan SE, Prager J, Villablanca JP, Farahani K, McBride DQ, Tsuruda JS, Morisoli B, Batzdorf $\mathrm{U}$, Johnson JP: Sciatica of nondisc origin and piriformis syndrome: Diagnosis by magnetic resonance neurography and interventional magnetic resonance imaging with outcome study of resulting treatment. J Neurosurg Spine 2:99-115, 2005

12. Fishman LM, Dombi GW, Michaelsen C, Ringel S, Rozbruch J, Rosner B, Weber C: Piriformis syndrome: Diagnosis, treatment, and outcome-a 10-year study. Arch Phys Med Rehabil 83:295-301, 2002

13. Fishman LM, Hosseini M: Piriformis syndrome - A diagnosis comes into its own. Muscle Nerve, 2019 (Epub ahead of print)

14. Fishman LM, Schaefer MP: The piriformis syndrome is underdiagnosed. Muscle Nerve 28:646-649, 2003

15. Foster MR: Piriformis syndrome. Orthopedics 25:821-825, 2002

16. Freiberg AH, Vinke TA: Sciatica and the sacroiliac joint. J Bone and Joint Surg 16:126-136, 1934 
17. Guvencer M, Akyer P, Iyem C, Tetik S, Naderi S: Anatomic considerations and the relationship between the piriformis muscle and the sciatic nerve. Surg Radiol Anat 30:467-474, 2008

18. Halpin RJ, Ganju A: Piriformis syndrome: A real pain in the buttock? Neurosurgery 65:A197-202, 2009

19. Hopayian K, Danielyan A: Four symptoms define the piriformis syndrome: An updated systematic review of its clinical features. Eur J Orthop Surg Traumatol 28:155-164, 2018

20. Hopayian K, Song F, Riera R, Sambandan S: The clinical features of the piriformis syndrome: A systematic review. Eur Spine J 19:2095-2109, 2010

21. Jankovic D, Peng P, van Zundert A: Brief review: Piriformis syndrome: Etiology, diagnosis, and management. Can J Anaesth 60:1003-1012, 2013

22. Kulcu DG, Naderi S: Differential diagnosis of intraspinal and extraspinal non-discogenic sciatica. J Clin Neurosci 15:12461252, 2008

23. Levin SM: Piriformis syndrome. Orthopedics 23:183-184, 2000

24. Md P, Rai R: Anatomical Study of Width and Thickness of Sciatic Nerve in the Gluteal Region, in $2^{\text {nd }}$ ISERD International Conference. Dubai, UAE, 2015:26-28

25. Michel F, Decavel P, Toussirot E, Tatu L, Aleton E, Monnier G, Garbuio P, Parratte B: Piriformis muscle syndrome: Diagnostic criteria and treatment of a monocentric series of 250 patients. Ann Phys Rehabil Med 56:371-383, 2013

26. Michel F, Decavel P, Toussirot E, Tatu L, Aleton E, Monnier G, Garbuio P, Parratte B: The piriformis muscle syndrome: An exploration of anatomical context, pathophysiological hypotheses and diagnostic criteria. Ann Phys Rehabil Med 56:300-311, 2013

27. Misirlioglu TO, Palamar D, Akgun K: Letter to the editor involving the article 'Piriformis muscle syndrome: A crosssectional imaging study in 116 patients and evaluation of therapeutic outcome'. Eur Radiol 28:5354-5355, 2018

28. Pace JB, Nagle D: Piriform syndrome. West J Med 124:435439, 1976

29. Palliyath S, Buday J: Sciatic nerve compression: Diagnostic value of electromyography and computerized tomography. Electromyogr Clin Neurophysiol 29:9-11, 1989
30. Papadopoulos EC, Khan SN: Piriformis syndrome and low back pain: A new classification and review of the literature. Orthop Clin North Am 35:65-71, 2004

31. Robinson DR: Pyriformis syndrome in relation to sciatic pain. Am J Surg 73:355-358, 1947

32. Siddiq MA, Hossain MS, Uddin MM, Jahan I, Khasru MR, Haider NM, Rasker JJ: Piriformis syndrome: A case series of 31 Bangladeshi people with literature review. Eur J Orthop Surg Traumatol 27:193-203, 2017

33. Silver JK, Leadbetter WB: Piriformis syndrome: Assessment of current practice and literature review. Orthopedics 21:11331135, 1998

34. Singh US, Meena RK, Singh CK, Singh AJ, Singh AM, Langshong R: Prevalence of piriformis syndrome among the cases of low back/buttock pain with sciatica: A prospective study. J Med Soc 27:94-99, 2013

35. Stewart JD: The piriformis syndrome is over diagnosed. Muscle Nerve 28:644-646, 2003

36. Stoll G, Bendszus M, Perez J, Pham M: Magnetic resonance imaging of the peripheral nervous system. J Neurol 256:10431051, 2009

37. Varenika V, Lutz AM, Beaulieu CF, Bucknor MD: Detection and prevalence of variant sciatic nerve anatomy in relation to the piriformis muscle on MRI. Skeletal Radiol 46:751-757, 2017

38. Vassalou EE, Katonis $\mathrm{P}$, Karantanas $\mathrm{AH}$ : Piriformis muscle syndrome: A cross-sectional imaging study in 116 patients and evaluation of therapeutic outcome. Eur Radiol 28:447458, 2018

39. Wada K, Hashimoto T, Miyagi R, Sakai T, Sairyo K: Diffusion tensor imaging and tractography of the sciatic nerve: Assessment of fractional anisotropy and apparent diffusion coefficient values relative to the piriformis muscle, a preliminary study. Skeletal Radiol 46:309-314, 2017

40. Yang HE, Park JH, Kim S: Usefulness of magnetic resonance neurography for diagnosis of piriformis muscle syndrome and verification of the effect after botulinum toxin type a injection: Two cases. Medicine (Baltimore) 94:e1504, 2015

41. Yeoman $W$ : The relation of arthritis of the sacro-iliac joint to sciatica, with an analysis of 100 cases. Lancet 2:1119-1122, 1928 